\title{
WENO Schemes for the Resolution of the Two-Dimensional Vlasov-Poisson System
}

\author{
Yacine Benhadid
}

\begin{abstract}
A numerical resolution of the two dimensional Vlasov-Poisson system is proposed. The time splitting method is used on the Vlasov equations to reduce the dimensions, then we apply the WENO (weighted essentially non-oscillatory) scheme based on the idea of Harten to solve our equation. We elaborate a transformation time-space to avoid the mixed problem and reducing the error. This algorithm is applied to the two dimensional Vlasov-Poisson system and an evolution of the density and the total energy is presented.
\end{abstract}

Index Terms-WENO schemes, vlasov equation, time splitting method.

\section{INTRODUCTION}

Various numerical methods have been developed for solving the Vlasov-Poisson equations. These methods can be parted in two main branches. The first is the well-known particle-in-cell (PIC) method [1], [2]. This one introduces the concept of pseudo-particles and relies on the computation of their trajectories. This method has been successfully used to simulate the behavior of collisionless laboratory and space plasmas and provides accurate results for modeling of large scale phenomena. Moreover suffer from intrinsic drawbacks.

An alternative approach based on an Eulerian approach consists in solving the Vlasov equation for the distribution function [3], [4]. Also an adaptative wavelets scheme is proposed in [5].

There is a large number of competing finite difference and related schemes for the solution of the Vlasov equation suggested in the literature; we have chosen to use the essentially nonoscillatory (ENO) schemes of Osher [6] and Osher and Shu [7] and the related weighed ENO (WENO) schemes Jiang and Peng [8]; Liu et al. [6]; Shu [7] for the following reasons: (1) stable schemes of arbitrarily high order accuracy exist, permitting accurate solutions on coarse grids (which is critical to the mesh refinement or coarsenment); (2) versions exist in any dimension so that we can extend our methodology to the three-dimensional case straightforwardly (see [9]).

The ENO scheme was successfully applied on the Vlasov equation in two dimensions [10].

In two dimensions, the Vlasov equation is written as follows [11] :

Manuscript received September 17, 2012; revised December 24, 2012. Yacine Benhadid is with the PAAET, Kuwait (email: y.benhadid@hotmail.com).

$$
\frac{\partial f}{\partial t}\left(t, x, y, v_{x}, v_{y}\right)+v_{x} \frac{\partial f}{\partial x}+v_{y} \frac{\partial f}{\partial y}+E_{x} \frac{\partial f}{\partial v_{x}}+E_{y} \frac{\partial f}{\partial v_{y}}=0
$$

We apply time splitting method [12] to the equation (1), we obtain then:

$$
\begin{gathered}
\frac{\partial f}{\partial t}+v_{x} \frac{\partial f}{\partial x}+v_{y} \frac{\partial f}{\partial y}=0 . \\
\frac{\partial f}{\partial t}+E_{x} \frac{\partial f}{\partial v_{x}}+E_{y} \frac{\partial f}{\partial v_{y}}=0 .
\end{gathered}
$$

From these equations, all work carry out until now were elaborate by again applying a stage of fractional steps to the equations (2) and (3). In this way, we find a problem in dimension one $(1 D)$ which we can solve successfully. But the time splitting method required, for the level of the numerical scheme, to cut the step of time by two to keep the same order of precision because it is necessary to symetrize the steps. With splitting again (2) et (3), we obtain:

$$
\left\{\begin{array} { l } 
{ ( A ) \frac { \partial f } { \partial t } + v _ { x } \frac { \partial f } { \partial x } = 0 , } \\
{ ( B ) \frac { \partial f } { \partial t } + v _ { y } \frac { \partial f } { \partial y } = 0 , }
\end{array} \quad \left\{\begin{array}{ll}
(C) \frac{\partial f}{\partial t}+E_{x} \frac{\partial f}{\partial v_{x}}=0, \\
(D) \frac{\partial f}{\partial t}+E_{y} \frac{\partial f}{\partial v_{y}}=0 .
\end{array}\right.\right.
$$

and the symmetric algorithm is now :

If we begin in time $t_{0}$, with $f\left(x, v, f(x ; v ; t=t 0)=f\left(t_{0}\right)\right.$ as initial condition, each solution of the equations $\mathrm{A}$ to $\mathrm{D}$ is used as an initial condition to the next step:

- Solution of (A) on a time $\Delta t / 2 \Rightarrow f_{1}\left(t_{0}+\Delta t / 2\right)$,

- Solution of (B) on a time $\Delta t / 2 \Rightarrow f_{2}\left(t_{0}+\Delta t / 2\right)$,

- Solution of (C) on a time $\Delta t / 2 \Rightarrow f_{3}\left(t_{0}+\Delta t / 2\right)$,

- Solution of (D) on a time $\Delta t \Rightarrow f_{4}\left(t_{0}+\Delta t\right)$,

- Solution of (C) on a time $\Delta t / 2 \Rightarrow f_{5}\left(t_{0}+\Delta t / 2\right)$,

- Solution of (B) on a time $\Delta t / 2 \Rightarrow f_{6}\left(t_{0}+\Delta t / 2\right)$,

- Solution of (A) on a time $\Delta t / 2 \Rightarrow f_{7}\left(t_{0}+\Delta t / 2\right)$,

In total 7 steps. A scheme non resplitted require only two steps. Also this algorithm is not adapted for large shifts which increase the error in particular by reducing the order of precision. This large shift is very realizable in our case and consequently any method used for the resolution of the equations results of the second splitting is not efficient and the results are rather erroneous and not very reliable. To 
avoid these errors, we established a method which reduced the error considerably.

The algorithm is to solve directly the equations $(1,2)$ and $(1,3)$ (without splitting) by using the idea of Harten [13] in two dimensions. In this case the large shift is not a constraint in our scheme because it represents a translation of our cell towards another. Then we will be able to take up the idea of splitting our equation but at this time the large shift was already carries out well on a complete step that the entire part first, then the small shift has to take into account for the continuation of the resolution. At this level we will use the method of the fractional steps (time splitting).

\section{WENO SCHEMES}

The basic idea of WENO, which is based on ENO [14] of course, is the following: instead of using only one of the candidate stencils to form the reconstruction, one uses a convex combination of all of them. So suppose the $k$ candidate stencils

$$
S_{r}(i)=x_{i r}, \ldots, x_{i r+k}, \quad r=0, \ldots, k-1
$$

produce $k$ different reconstructions to the value $v_{i+1 / 2}$, according to (5),

$$
v_{i+\frac{1}{2}}^{(r)}=\sum_{j=0}^{k-1} c_{r j} \bar{v}_{i-r+j}, \quad r=0, \ldots, k-1
$$

WENO reconstruction would take a convex combination of all $v_{i+1 / 2}^{(r)}$ defined in (6) as a new approximation to the cell boundary value $v\left(x_{i+1 / 2}\right)$ :

$$
v_{i+\frac{1}{2}}=\sum_{j=0}^{k-1} w_{r} v_{i+1 / 2}^{(r)}
$$

We require

$$
w_{r} \geq 0, \quad \sum_{j=0}^{k-1} w_{r}=1
$$

for stability and consistency.

If the function $v(x)$ is smooth in all of the candidate stencils (5), there are constants $d_{r}$ such that

$$
v_{i+\frac{1}{2}}=\sum_{j=0}^{k-1} d_{r} v_{i+1 / 2}^{(r)}=v\left(x_{i+1 / 2}\right)+o\left(\Delta x^{2 k-1}\right)
$$

and for the other

$$
v_{i-\frac{1}{2}}=\sum_{j=0}^{k-1} \tilde{d}_{r} v_{i+1 / 2}^{(r)}=v\left(x_{i-1 / 2}\right)+o\left(\Delta x^{2 k-1}\right),
$$

We have by symmetry

$$
\tilde{d}_{r}=d_{k-1-r}
$$

For example, $d_{r}$ for $1 \leq k \leq 3$ are given by

$$
\begin{aligned}
d_{0}=1, & k=1 ; & \\
d_{0}=\frac{2}{3}, & d_{1}=\frac{1}{3}, & k=2 \\
d_{0}=\frac{3}{10}, & d_{1}=\frac{3}{5}, & d_{2}=\frac{1}{10}, \quad k=3
\end{aligned}
$$

We can see that $d_{r}$ is always positive and, due to constancy,

$$
\sum_{r=0}^{k-1} d_{r}=1
$$

The weights should be smooth functions of the all averages involved. In fact, the weights described below are $C l$. We would like to have weights which are computationally efficient. Thus, polynomials or rational functions are preferred over exponential type functions.

All the considerations lead to the following form of weights:

$$
w_{r}=\frac{\alpha_{r}}{\sum_{s=0}^{k-1} \alpha_{s}}, \quad r=0, \ldots, k-1
$$

with

$$
\alpha_{r}=\frac{d_{r}}{\left(\varepsilon+\beta_{r}\right)^{2}}
$$

Here $\varepsilon>0$ is introduced to avoid the denominator to become 0 and $\beta_{r}$ is a smoothness measurement of the flux function on the $k$-th candidate stencil.

Consideration for a smooth flux and for the role of higher order variations leads us to the following measurement for the smoothness: let the reconstruction polynomial on the stencil $S_{r}(i)$ be denoted by $p_{r}(x)$, we define:

$$
\beta_{r}=\sum_{l=0}^{k-1} \int_{x_{i-1 / 2}}^{x_{i+1 / 2}} \Delta x^{2 l-1}\left(\frac{\partial^{l} p_{r}(x)}{\partial^{l} x}\right) d x .
$$

The right hand side is just a sum of the squares of scaled $L^{2}$ norms for all the derivatives of the interpolation polynomial $p_{r}(x)$ over the interval $\left(x_{i-1 / 2}, x_{i+1 / 2}\right)$. The factor $\Delta x^{2 l-1}$ is introduced to remove any $\Delta x$ dependency in the derivatives.

For example, when $k=2$

$$
\left\{\begin{array}{l}
\beta_{0}=\left(\bar{v}_{i+1}-\bar{v}_{i}\right)^{2}, \\
\beta_{1}=\left(\bar{v}_{i}-\bar{v}_{i-1}\right)^{2} .
\end{array}\right.
$$


and for $k=3$, (61) gives:

$$
\left\{\begin{array}{c}
\beta_{0}=\frac{13}{12}\left(\bar{v}_{i}-2 \bar{v}_{i+1}-\bar{v}_{i+2}\right)^{2}+\frac{1}{4}\left(3 \bar{v}_{i}-4 \bar{v}_{i+1}-\bar{v}_{i+2}\right)^{2}, \\
\beta_{1}=\frac{13}{12}\left(\bar{v}_{i-1}-2 \bar{v}_{i}-\bar{v}_{i+1}\right)^{2}+\frac{1}{4}\left(\bar{v}_{i-1}-\bar{v}_{i+1}\right)^{2}, \\
\beta_{2}=\frac{13}{12}\left(\bar{v}_{i-2}-2 \bar{v}_{i-1}+\bar{v}_{i}\right)^{2}+\frac{1}{4}\left(\bar{v}_{i-2}-4 \bar{v}_{i-1}+3 \bar{v}_{i}\right)^{2}
\end{array}\right.
$$

Now, we can form the weights $w_{r}$ and $\bar{w}_{r}$ using (8) and

$$
\tilde{w}_{r}=\frac{\alpha_{r}}{\sum_{s=0}^{k-1} \tilde{\alpha}_{s}}, \quad \tilde{\alpha}_{r}=\frac{\tilde{d}_{r}}{\left(\varepsilon+\beta_{r}\right)^{2}}
$$

Finally, we can find the (2k-1)-th order reconstruction

$$
\begin{aligned}
& v_{i+\frac{1}{2}}^{+}=\sum_{j=0}^{k-1} w_{r} v_{i+1 / 2}^{(r)} \\
& v_{i-\frac{1}{2}}^{-}=\sum_{j=0}^{k-1} \tilde{w}_{r} v_{i-1 / 2}^{(r)}
\end{aligned}
$$

And now, we can return to the monotone flux chosen before for the ENO schemes then follows all the steps as in it (use (9) to compute the flux then form the scheme ).

\section{TIME-SPACE TRANSITION}

Our problem is to carry out a passage of the integral in time $t$ to an integral in space $x$ or $y$. For that, we return to dimension $1(1 d)$ while making our calculations compared to the transport equation:

$$
\frac{\partial f}{\partial t}+a \frac{\partial f}{\partial x}=0
$$

By integrating, we obtain

$$
\int_{t}^{t+\Delta t} a f(\tau, x) d \tau=-\int_{y_{1}}^{y_{2}} f(p) d p
$$

In our case, $a$ is a constant consider a function $X(t 0 ; t ; x)$ such:

$$
f(t, x)=f\left(X\left(t_{0}, t, x\right)\right) \frac{\partial X}{\partial x}\left(t_{0}, t, x\right)
$$

which satisfied

$$
\frac{\partial X}{\partial t}\left(t_{0}, t, x\right)+a \frac{\partial X}{\partial x}\left(t_{0}, t, x\right)=0,
$$

with:

$$
\left\{\begin{array}{l}
\frac{\partial X}{\partial x}\left(t_{0}, t, x\right)=-a \\
X\left(t_{0}, n t_{0}, x\right)=x
\end{array}\right.
$$

Taking $a$ constant gives:

$$
X\left(t_{0}, t, x\right)=x-a\left(t-t_{0}\right) .
$$

We introduce the relation now (11) in the integral in time, we obtain then:

$$
\int_{t}^{t+\Delta t} a f(\tau, X) d \tau=\int_{t}^{t+\Delta t} a f\left(X\left(t_{0}, t, x\right)\right) \frac{\partial X}{\partial x}\left(t_{0}, t, x\right) d t .
$$

from (3.34), we have

$$
\int_{t}^{t+\Delta t} a f(\tau, X) d \tau=-\int_{t}^{t+\Delta t} f(X) \frac{\partial X}{\partial x} d t
$$

Putting $X=z$ and with the conditions (12), the equation (13) becomes

$$
\int_{t}^{t+\Delta t} a f(\tau, X) d \tau=-\int_{x t}^{X(t, t+\Delta t, x)} f(Z) d Z
$$

We obtain then desired transformation which transforms a integral into time in another in space.

We will use the transformation (14) to calculate $\hat{f}_{i+1 / 2, j}$ and $\hat{g}_{i, j+1 / 2}$ and by similarity $\hat{f}_{i-1 / 2, j}$ and $\hat{g}_{i, j-1 / 2}$.

\section{APPLiCATION to the Vlasov-POISSON SYSTEM}

Consider

$$
\hat{f}_{i+1 / 2, j}=\frac{1}{\tau \Delta y} \int_{o}^{\tau} \int_{-\Delta y / 2}^{\Delta y / 2} f\left(u\left(x_{i+1 / 2}, y_{j}+\eta, t+t_{n}\right)\right) d \eta d t
$$

In the case of the equation of Vlasov, we have:

$$
f(u)=v_{x} u \text {. }
$$

There are several cases to study carrying out our calculations for a small shift using finite difference method.

Case 1: $\quad \frac{\Delta x}{2}-\alpha \leq \xi \leq \frac{\Delta x}{2}$, and $-\frac{\Delta y}{2} \leq \eta \leq \frac{\Delta y}{2}$ with $0 \leq \alpha \leq \frac{\Delta x}{2}$

$$
\begin{gathered}
\int_{0}^{\tau} \int_{-\Delta y / 2}^{\Delta y / 2} f\left(u\left(x_{i+1 / 2}, y_{j}+\eta, t+t_{n}\right) d \eta d t=\right. \\
\int_{0}^{\tau} \int_{-\Delta y / 2}^{\Delta y / 2} v_{x} u\left(x_{i+1 / 2}, y_{j}+\eta, t+t_{n}\right) d \eta d t
\end{gathered}
$$

By using the transformation (14), we obtain then: 


$$
\begin{aligned}
A=\int_{0}^{\tau} \int_{-\Delta y / 2}^{\Delta y / 2} v_{x} u\left(x_{i+1 / 2}, y_{j}+\eta, t+t_{n}\right) d \eta d t= \\
\\
\quad \int_{\Delta x / 2-\alpha-\Delta y / 2}^{\Delta x / 2} \int_{x}^{\Delta y / 2} v_{x} u\left(x_{i}+\xi, y_{j}+\eta, t+t_{n}\right) d \eta d t
\end{aligned}
$$

Using five points finite difference, we develop our function $u$ such as:

$$
\begin{aligned}
u\left(x_{i}+\xi, y_{j}\right. & \eta)=u_{i, j}+\frac{\xi}{2 \Delta x}\left(u_{i+1, j}-u_{i-1, j}\right) \\
& +\frac{\eta}{2 \Delta y}\left(u_{i, j+1}-u_{i, j-1}\right)+\frac{\xi \eta}{4 \Delta x \Delta y}\left(u_{i+1, j+1}-u_{i-1, j+1}-u_{i+1, j-1}+u_{i-1, j-1}\right) .
\end{aligned}
$$

Then $A$ becomes:

$$
\begin{aligned}
A=- & \int_{\Delta x / 2-\alpha-\Delta y / 2}^{\Delta x / 2} \int^{\Delta y / 2}\left[u\left(x_{i}+\xi, y_{j}+\eta\right)=u_{i, j}+\frac{\xi}{2 \Delta x}\left(u_{i+1, j}-u_{i-1, j}\right)\right. \\
& \left.+\frac{\eta}{2 \Delta y}\left(u_{i, j+1}-u_{i, j-1}\right)+\frac{\xi \eta}{4 \Delta x \Delta y}\left(u_{i+1, j+1}-u_{i-1, j+1}-u_{i+1, j-1}+u_{i-1, j-1}\right)\right] d \eta d \xi .
\end{aligned}
$$

$$
\begin{gathered}
A=-\int_{\Delta x / 2-\alpha}^{\Delta x / 2}\left[\Delta y u_{i j}+\frac{\xi \Delta y}{2 \Delta x}\left(u_{i+1, j}-u_{i-1, j}\right)\right] d \xi \\
A=-\Delta y \alpha u_{i j}+\frac{\left(\alpha \Delta x-\alpha^{2}\right) \Delta y}{4 \Delta x}\left(u_{i+1, j}-u_{i-1, j}\right) .
\end{gathered}
$$

Case 2: $-\frac{\Delta x}{2} \leq \xi \leq-\frac{\Delta x}{2}+\alpha,{ }^{\text {and }}-\frac{\Delta y}{2} \leq \eta \leq \frac{\Delta y}{2}$ with $\mathrm{O} \leq \alpha \leq \frac{\Delta x}{2}: \hat{f}_{i-1 / 2, j}$ is written as:

$$
\hat{f}_{i-1 / 2, j}=\frac{1}{\tau \Delta y} \int_{o}^{\tau} \int_{-\Delta y / 2}^{\Delta y / 2} f\left(u\left(x_{i-1 / 2}, y_{j}+\eta, t+t_{n}\right)\right) d \eta d t
$$

Following the same calculations of the first case and now denoted by $B$, we have:

$$
B=-\Delta y \alpha u_{i j}+\frac{\left(\alpha^{2}-\alpha \Delta x\right) \Delta y}{4 \Delta x}\left(u_{i+1, j}-u_{i-1, j}\right)
$$

Let us compute $\hat{g}_{i, j+1 / 2}$ and $\hat{g}_{i, j-1 / 2}$.It is carried out according to the technique of previous description:

$$
\hat{g}_{i, j+1 / 2}=\frac{1}{\tau \Delta x} \int_{o}^{\tau} \int_{-\Delta x / 2}^{\Delta x / 2} v_{y} u\left(x_{i}+\xi, y_{j+1 / 2}, t+t_{n}\right) d \xi d t
$$

Case 3: $-\frac{\Delta x}{2} \leq \xi \leq \frac{\Delta x}{2}$, and $\frac{\Delta y}{2}-\beta \leq \eta \leq \frac{\Delta y}{2}$ with $0 \leq \beta \leq \frac{\Delta y}{2}:$

while using (14), there is

$$
\hat{g}_{i, j+1 / 2}=\frac{1}{\tau \Delta x} \int_{\Delta y / 2-\beta}^{\Delta y / 2} \int_{-\Delta x / 2}^{\Delta x / 2} v_{y} u\left(x_{i}+\xi, y_{j+1 / 2}, t+t_{n}\right) d \xi d t
$$

We note by $C$ the following integral:

$$
C=\int_{\Delta x / 2-\alpha}^{\Delta x / 2} \int_{-\Delta y / 2}^{\Delta y / 2} v_{x} u\left(x_{i}+\xi, y_{j}+\eta, t+t_{n}\right) d \eta d \xi
$$

In the same manner, we obtain:

$$
C=-\Delta x \beta u_{i j}+\frac{\left(\beta \Delta x-\beta^{2}\right) \Delta y}{4 \Delta y}\left(u_{i, j+1}-u_{i, j-1}\right) .
$$

Case 4: $-\frac{\Delta x}{2} \leq \xi \leq \frac{\Delta x}{2}$, and $-\frac{\Delta y}{2} \leq \eta \leq-\frac{\Delta y}{2}+\beta$ with $\mathrm{O} \leq \beta \leq \frac{\Delta y}{2}$

After same calculation for $\hat{g}_{i, j-1 / 2}$, we find the integral $D$ :

$$
D=-\Delta x \beta u_{i j}+\frac{\left(\beta^{2}-\beta \Delta x\right) \Delta y}{4 \Delta y}\left(u_{i, j+1}-u_{i, j-1}\right) .
$$

The general relation (4.40) become:

$$
\begin{aligned}
\overline{\bar{f}}_{n+1}= & \overline{\bar{f}}_{n}-\lambda_{x}\left[\frac{\left(\alpha \Delta x-\alpha^{2}\right) \Delta y}{4 \Delta x}\left(u_{i+1, j}-u_{i-1, j}\right)\right] \\
& -\lambda_{y}\left[\frac{\left(\beta \Delta x-\beta^{2}\right) \Delta y}{4 \Delta y}\left(u_{i, j+1}-u_{i, j-1}\right)\right] .
\end{aligned}
$$

Considering the variations of the shift in space, we find new boundaries.

$$
\begin{aligned}
\overline{\bar{f}}_{i j}^{n+1} & =\overline{\bar{f}}_{i j}^{n}-\frac{1}{\Delta x \Delta y}\left[\int_{x_{i+1 / 2}-\alpha}^{x_{i+1 / 2}} \int_{y_{j-1 / 2}}^{y_{j+1 / 2}} f d x d y\right. \\
& -\int_{x_{i+1 / 2}-\alpha}^{x_{i+1 / 2}} \int_{y_{j-1 / 2}}^{y_{j+1 / 2}} f d x d y+\int_{x_{i+1 / 2}-\alpha}^{x_{i+1 / 2}} \int_{y_{j-1 / 2}}^{y_{j+1 / 2}} f d x d y \\
& \left.-\int_{x_{i+1 / 2}-\alpha}^{x_{i+1 / 2}} \int_{y_{j-1 / 2}}^{y_{j+1 / 2}} f d x d y\right]
\end{aligned}
$$

Hence, we obtain then:

$$
\begin{aligned}
\overline{\bar{f}}_{i j}^{n+1} & =\overline{\bar{f}}_{i j}^{n}+\left(\frac{3}{4}\left(\frac{\alpha}{\Delta x}+\frac{\beta}{\Delta y}\right)-\frac{1}{4}\left(\frac{\alpha}{\Delta x}\right)^{2}-\frac{1}{4}\left(\frac{\beta}{\Delta y}\right)^{2}\right) f_{i j} \\
+ & \left(\frac{1}{4} \frac{\alpha}{\Delta x}-\frac{1}{4}\left(\frac{\alpha}{\Delta x}\right)^{2}\right) f_{i+1, j}-\left(\frac{1}{4} \frac{\alpha}{\Delta x}+\frac{1}{4}\left(\frac{\alpha}{\Delta x}\right)^{2}\right) f_{i-1, j} \\
+ & \left(\frac{1}{4} \frac{\alpha}{\Delta x}+\frac{1}{4}\left(\frac{\alpha}{\Delta x}\right)^{2}\right) f_{i-2, j}+\left(\frac{1}{4} \frac{\beta}{\Delta y}-\frac{1}{4}\left(\frac{\beta}{\Delta y}\right)^{2}\right) f_{i, j+1} \\
& -\left(\frac{1}{4} \frac{\beta}{\Delta y}-\frac{1}{4}\left(\frac{\beta}{\Delta y}\right)^{2}\right) f_{i, j-1}-\left(\frac{1}{4} \frac{\beta}{\Delta y}-\frac{1}{4}\left(\frac{\beta}{\Delta y}\right)^{2}\right) f_{i, j-2} .
\end{aligned}
$$

We start the simulation from a two stream situation, both 
in $x$ and $y$, with a perturbation term:

$$
f\left(t=0, x, y, v_{x}, v_{y}\right)=\frac{1}{2 \pi} e^{-\left(v_{x}^{2}+v_{y}^{2}\right) / 2}(1+\text { pert }),
$$

where

$$
\text { pert }=0.02[k \cos (x)+k \cos y] \text {. }
$$

The initial condition is Maxwellian in v-space and with a cosine perturbation in $\mathrm{x}$ and $\mathrm{y}$ space.

As shown in Fig. 1, the initial perturbation which has been introduced in the distribution function make some noise on the Vlasov model but using splitting scheme we contain this noise to be stable in sense of increasing. Both density and total energy are shown in Fig. 1 varying with time evolution.
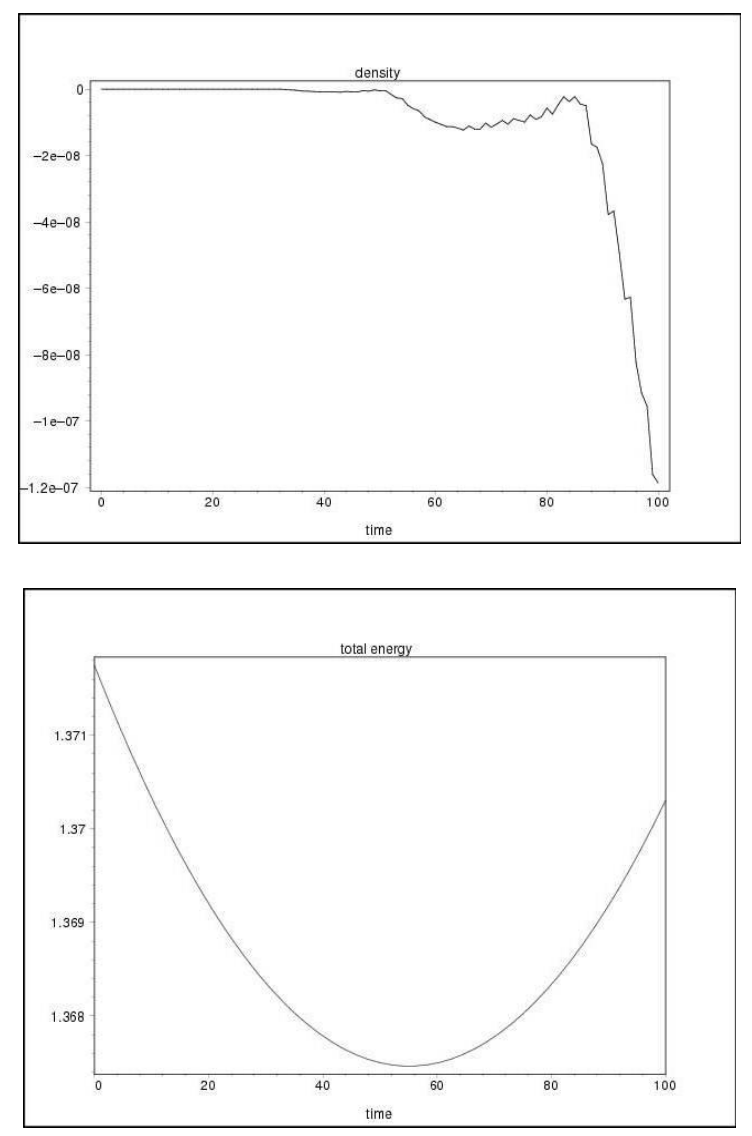

Fig. 1. Time evolution of the mean normalized density and the total energy.

\section{CONCLUSION}

We have presented the WENO method based on the idea of Harten and its application to solve the two-dimensional Vlasov-Poisson system. By splitting Vlasov equation only one time, we reduce the number of steps.

Our algorithm is applied on the first splitted equation, then we determine the $E$ from Poisson equation and finally we solve the second splitted equation using the same shift.

We have separated integer part and left fractional in elaborating the boundaries of the integrals. The method used here is faster than other method [2], [15], but we obtain a similar results.

\section{REFERENCES}

[1] Armstrong, "PIC codes," J. Comp. Phys., vol. 2, 1976.

[2] C. K. Bridsall and A. B. Langdon, Plasma physics via computer simulation, Mc graw-hill, 1980.

[3] B. Cockburn, C. Johnson, C. W. Shu, and E. Tadmor, Advanced Numerical Approximation of nonlinear Hyperbolic Equations, Springer, pp. 25, 1997.

[4] E. Godlewski and P. A. Raviart, Numerical approximation of hyperbolic systems of conservation laws, Springer, pp. 37, 1996.

[5] Y. Benhadid, An adaptative Wavelets methods for solving the two-dimensional Vlasov equation, IEEE press, AEM2011, pp. 83-87, 2011.

[6] X. D. Liu, S. Osher, and T. Shan, "Weighted Essentially Nonoscillatory shemes," Journal of Computational Physics, vol. 115, no. 1, pp. 200-212, 1994.

[7] C. W. Shu, "Essentially Non-Oscillatory and Weighted Essentially Non-Osciallatory Schemes for Hyperbolic Conservation Laws," ICASE, vol. $65,1997$.

[8] G. S. Jiang and C. W. Shu, "Efficient implementation of weighted ENO schemes," Journal of Computational Physics, vol. 126, no. 1, pp. 202-228, 1996.

[9] C. Q. Hu and C. W. Shu, "Weighted Essentially Non-Oscillatory schemes on triangular meshes," Journal of Computational Physics, vol 150, no. 1, pp. 97-127, 1999.

[10] Y. Benhadid, "ENO schemes for the two dimensional Vlasov Equation," IEEE press, ICSPS2009, pp. 942-947, 2009.

[11] Dobrushin, Vlasov equation, Functional Analysis, vol. 13, pp. 115-123, 1979.

[12] N. Yanenko, "The methods of Fractionals steps," Verlag, Springer, 1971.

[13] A. Harten, B, Engquist, S. Osher, and S. Chakravarthy, "Uniformly high-order essentially non-oscillatory schemes," Applied Numerical Mathematics, vol. 24, 1987.

[14] S. Osher and C. W. Shu, "Efficient implementation of ENO schemes," JCP, vol. 26, 1989.

[15] M. L. Begue, A. Guizzo, and P. Bertrand, "Two dimensional Vlasov simulation of Raman scattering and plasma beatwave acceleration on parallel computers," J. Comp. Phys., vol. 151, pp. 458-478, 1999.

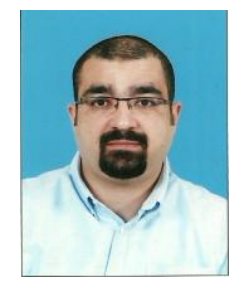

Yacine Benhadid earned the B.S. degree in mathematics at Badji Mokhtar University, Annaba, Algeria, in 1994, M.S. degree in applied mathematics at the university of Tours-Orleans, France, in 1995, and the Ph.D. degree in applied mathematics at the university of Orleans, in 2000 . He is currently an assistant professor of mathematics in the Department of Mathematics at PAAET, Kuwait. His research interests include numerical analysis, optimal control, wavelets, simulation and optimization. He has several publications in journals of mathematics, operations research and computational physics. 